Received: 4 December 2017

Accepted: 13 April 2018

Published online: 02 May 2018

\section{OPEN Ultrafast magnetization modulation induced by the electric field component of a terahertz pulse in a ferromagnetic-
semiconductor thin film}

\author{
Tomoaki Ishii ${ }^{1}$, HiromichiYamakawa ${ }^{2}$, Toshiki Kanaki ${ }^{1}$, Tatsuya Miyamoto ${ }^{2}$, Noriaki Kida ${ }^{2}$, \\ Hiroshi Okamoto ${ }^{2}$, Masaaki Tanaka ${ }^{1,3}$ \& Shinobu Ohya ${ }^{1,3,4}$
}

High-speed magnetization control of ferromagnetic films using light pulses is attracting considerable attention and is increasingly important for the development of spintronic devices. Irradiation with a nearly monocyclic terahertz pulse, which can induce strong electromagnetic fields in ferromagnetic films within an extremely short time of less than $\sim 1$ ps, is promising for damping-free high-speed coherent control of the magnetization. Here, we successfully observe a terahertz response in a ferromagnetic-semiconductor thin film. In addition, we find that a similar terahertz response is observed even in a non-magnetic semiconductor and reveal that the electric-field component of the terahertz pulse plays a crucial role in the magnetization response through the spin-carrier interactions in a ferromagnetic-semiconductor thin film. Our findings will provide new guidelines for designing materials suitable for ultrafast magnetization reversal.

In ferromagnetic materials, typically more than a few hundred picoseconds are necessary to reverse the magnetization when using spin-transfer torque or light irradiation ${ }^{1-13}$, limiting the operational speed of magnetic memory devices. Meanwhile, using a terahertz light pulse, a strong electromagnetic field can be induced within an extremely short time of less than $\sim 1$ picosecond in ferromagnetic thin films, in which the spin-lattice relaxation is too slow to follow the electromagnetic fields ${ }^{11}$. Thus, terahertz pulse control of the magnetization is promising for ultrafast magnetization reversal within a few picoseconds. In the previous studies on terahertz pump-probe measurements, a tiny modulation in the magnetization by a terahertz pulse was demonstrated for ferromagnetic-metal thin films such as $\mathrm{Co}, \mathrm{Ni}, \mathrm{Fe}$ and $\mathrm{CoFeB}^{11-13}$. Until now, the origin of this phenomenon has been attributed to the Landau-Lifschitz-Gilbert (LLG) torque ${ }^{11-13}$, which is induced by the magnetic-field component of the terahertz pulse, and to the demagnetization caused by the heating ${ }^{11,13}$

Recently, static-electric-field control of the magnetization vector ${ }^{14-16}$, Curie temperature and coercivity ${ }^{17,18}$ has been reported for magnetic metal thin films; however, thus far, the electric-field component of the terahertz pulse has not often been associated with the magnetization modulation ${ }^{13}$. In non-magnetic materials, optical properties are known to be influenced by the modulation of the spatial carrier distribution induced by the electric field of light ${ }^{19-21}$, which is called the Franz-Keldysh effect (FKE). The FKE has been investigated mainly for semiconductors rather than metals because semiconductors have a low carrier density and are sensitive to electric fields. GaAs is a suitable semiconductor for our study because it becomes ferromagnetic when doped with Mn and because $\mathrm{Mn}$-doped GaAs (GaMnAs) is fairly sensitive to an external optical stimulus ${ }^{5,22}$. Comparing GaAs samples doped

${ }^{1}$ Department of Electrical Engineering and Information Systems, The University of Tokyo, 7-3-1 Hongo, Bunkyo-ku, Tokyo, 113-8656, Japan. ²Department of Advanced Materials Science, Graduate School of Frontier Sciences, The University of Tokyo, Chiba, 277-8561, Japan. ${ }^{3}$ Center for Spintronics Research Network, Graduate School of Engineering, The University of Tokyo, 7-3-1 Hongo, Bunkyo-ku, Tokyo, 113-8656, Japan. ${ }^{4}$ Institute of Engineering Innovation, Graduate School of Engineering, The University of Tokyo, 7-3-1 Hongo, Bunkyo-ku, Tokyo, 113-8656, Japan. Correspondence and requests for materials should be addressed to T.I. (email: ishii@cryst.t.u-tokyo.ac.jp) or M.T. (email: masaaki@ee.t.u-tokyo.ac.jp) or S.O. (email: ohya@cryst.t.u-tokyo.ac.jp) 
a

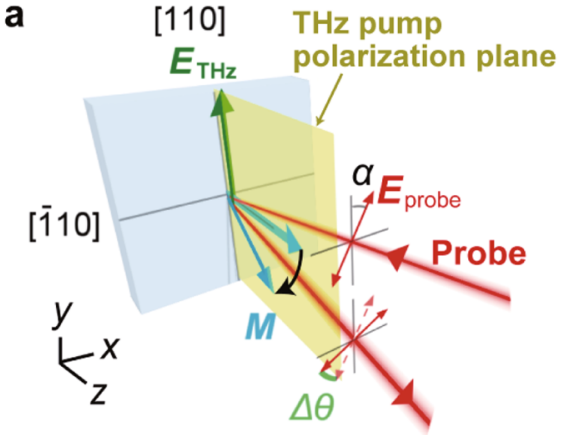

C

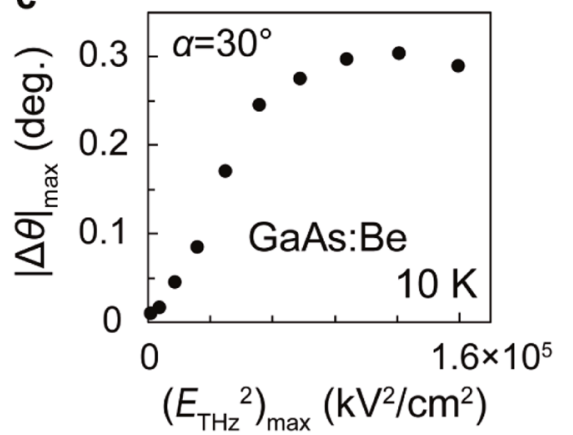

b
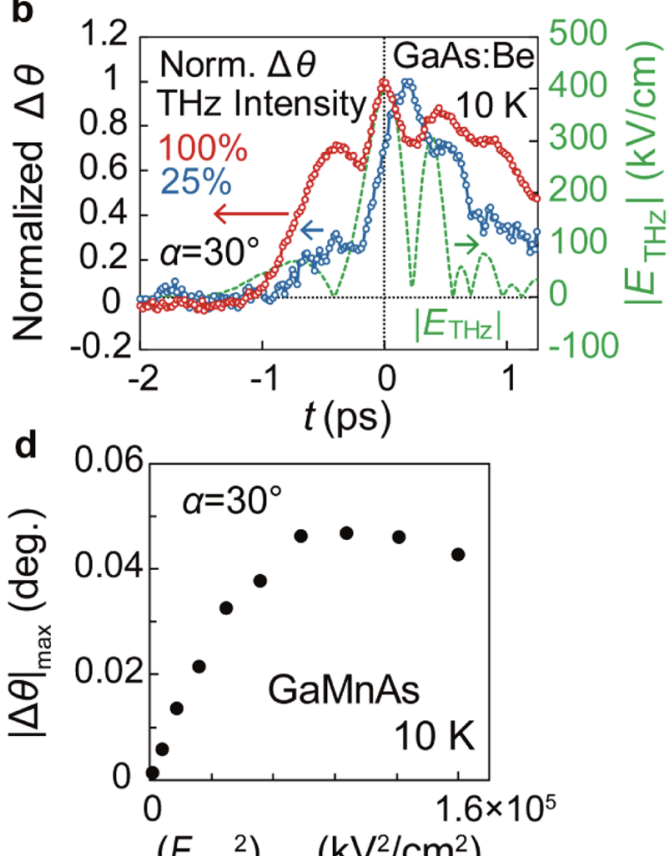

Figure 1. Overview of the experiment and the observation of $\Delta \theta$ induced by the Franz-Keldysh effect. (a) Schematic illustration of the experimental setup. The terahertz pump pulse (yellow) is focused on the GaAs:Be or GaMnAs sample surface, and the following probe pulse with a delay time $t$ detects the excited dynamics. Both the pump and probe pulses are linearly polarized. The strong terahertz-pump pulse with a centred frequency of $1 \mathrm{THz}$, whose electric field $E_{\mathrm{THz}}$ is along the [110] axis, is generated by optical rectification using a $\mathrm{LiNbO}_{3}$ crystal. The incident angle of the probe pulse is tilted by $10^{\circ}$ from the sample normal towards the in-plane [110] axis. The magnetization (pale blue arrow) is tilted from the sample normal by the terahertz pump pulse. The angle of the probe polarization plane from the [110] axis towards the [110] axis is defined as $\alpha$. (b) Time evolution of $\Delta \theta$ (red circles) measured at $10 \mathrm{~K}$ without an external magnetic field for GaAs:Be when applying $E_{\mathrm{THz}}$ expressed by the green dotted curve. $\Delta \theta$ is normalized by its maximum value. The blue circles express $\Delta \theta$ when the intensity $E_{\mathrm{THz}}{ }^{2}$ of the terahertz pump pulse is $25 \%$ of the green dotted curve (i.e., the maximum $E_{\mathrm{THz}}$ is $200 \mathrm{kV} / \mathrm{cm}$ ). $\alpha$ is set to $30^{\circ}$. (c,d) Maximum value of the time evolution of $|\Delta \theta|$ obtained for $\mathrm{GaAs:Be}(\mathbf{c})$ and $\mathrm{GaMnAs}(\mathbf{d})$ plotted as a function of the maximum value of $E_{\mathrm{THz}}{ }^{2}$ at $10 \mathrm{~K}$. These measurements are carried out without an external magnetic field when $\alpha$ is set to $30^{\circ}$.

with non-magnetic atoms and with $\mathrm{Mn}$ atoms can demonstrate how the electric field of the terahertz pulse influences the magnetization in ferromagnetic GaMnAs.

\section{Results}

Samples and experimental setup. In our terahertz pump-probe measurements, we used thin films so that the terahertz pump beam can penetrate them. We use a non-magnetic semiconductor Be-doped GaAs thin film (referred to as GaAs:Be, Be acceptor concentration: $10^{19} \mathrm{~cm}^{-3}$, thickness: $40 \mathrm{~nm}$ ) and a ferromagnetic semiconductor $\mathrm{Ga}_{0.94} \mathrm{Mn}_{0.06}$ As thin film (thickness: $20 \mathrm{~nm}$ ) with a perpendicular easy magnetization axis (see Methods). We utilize strong terahertz-pump pulses $(\sim 400 \mathrm{kV} / \mathrm{cm})$ with the centered frequency of $1 \mathrm{THz}$, whose electric field $E_{\mathrm{THz}}$ is aligned along the [110] axis in the film plane (Fig. 1a). We detect the change $\Delta \theta$ in the polarization rotation of the reflected probe pulse with a delay time $t$ relative to the terahertz-pump pulse. All measurements are carried out at $10 \mathrm{~K}$ (see Methods).

Observation of $\Delta \boldsymbol{\theta}$ induced by the FKE. Here, we show that the strong FKE is induced by the terahertz pump pulses both in GaAs:Be and GaMnAs. The FKE induces magnetization modulation and birefringence. In the following discussion, the subscript "max" refers to the maximum value of the transient. In GaAs:Be, evidence of the FKE can be seen in the $\left(E_{\mathrm{THz}}\right)_{\max }$ dependence of $(-\Delta R / R)_{\max }$ and in the time evolution of $-\Delta R / R$ (Supplementary Information Fig. S1); $(-\Delta R / R)_{\max }$ increases and saturates with increasing $\left(E_{\mathrm{THz}}{ }^{2}\right)_{\max }$ and the peak positions of $-\Delta R / R$ depend on $\left.\left(E_{\mathrm{THz}}\right)^{2}\right)_{\max }$ (see Supplementary Information Sec. A). These are distinctive features of the $\mathrm{FKE}^{23}$. Owing to the polarization of the terahertz pulse along [110], the FKE induces the anisotropy of the reflectivities between [110] and [1ㅣㅣ polarized light beams ${ }^{24}$. This anisotropy leads to the birefringence and thus the polarization rotation. These effects are actually observed in non-magnetic GaAs:Be, as shown in Fig. 1b, where the angle $\alpha$ between the electric field vector $E_{\text {probe }}$ of the probe and $E_{\mathrm{THz}}(/ /[110])$ is set to $30^{\circ}$ (Fig. 1a). As shown in Fig. 1c, $|\Delta \theta|_{\max }$ tends to saturate as $\left(E_{\mathrm{THz}}{ }^{2}\right)_{\max }$ increases, confirming that the observed $\Delta \theta$ is induced by the $\mathrm{FKE}^{23}$. For GaMnAs, we see that similar saturating behaviour appears in the $\left(E_{\mathrm{THz}}{ }^{2}\right)_{\max }$ dependence of $|\Delta \theta|_{\max }$ (Fig. 1d). Here, $\alpha$ is also set to $30^{\circ}$. This result indicates that $\Delta \theta$ is mainly attributed to the birefringence due to the 


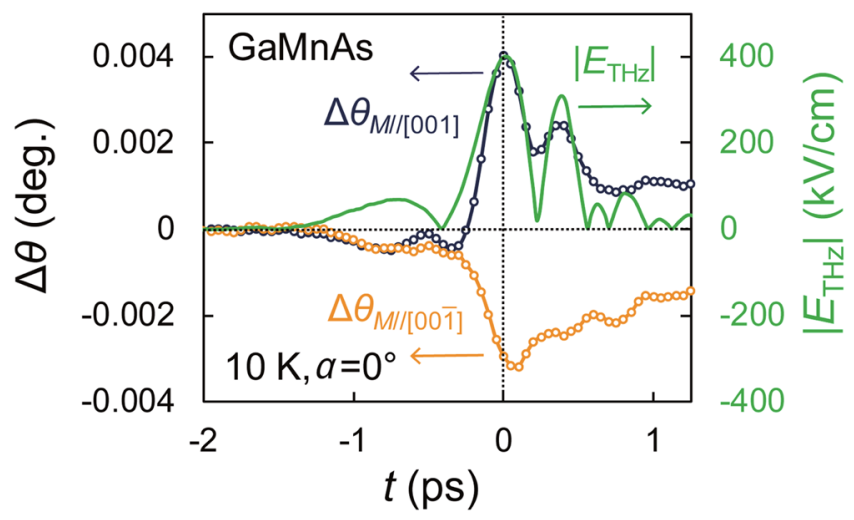

Figure 2. Terahertz response of the GaMnAs film. Blue and orange open circles represent $\Delta \theta$ measured at $10 \mathrm{~K}$ for the $\mathrm{Ga}_{0.94} \mathrm{Mn}_{0.06} \mathrm{As}$ thin film when $E_{\text {probe }} / / E_{\mathrm{THz}}\left(\alpha=0^{\circ}\right)$ with the magnetic field of $30 \mathrm{mT}$ is applied in the $[001]$ and [001] directions, respectively. $\left|E_{\mathrm{THz}}\right|$ is shown by the green solid curve.

FKE. The $\Delta \theta$ value observed for GaMnAs is smaller than that for GaAs:Be because the carrier density of GaMnAs is larger than that of GaAs:Be. Note that the small drop of $|\Delta \theta|_{\max }$ at high electric fields is due to the shift of the absorption edge by the $\mathrm{FKE}^{25}$. Because the signal induced by the birefringence is not directly related to the magnetization dynamics, this component should be removed.

Coherent magnetization modulation by $E_{\mathrm{THz}}$. By setting $E_{\text {probe }} / / E_{\mathrm{THz}}(/ /[110])$ (i.e., $\alpha=0^{\circ}$ ), the effect of the birefringence can be minimized, and the magnetic signal becomes dominant (Supplementary Information Sec. B). In fact, the sign of $\Delta \theta$ is almost perfectly inverted when the magnetic field $(=30 \mathrm{mT})$, which is applied perpendicular to the sample surface to align the magnetization, is reversed (orange and blue circles in Fig. 2). This means that the observed signal in Fig. 2 is purely a magnetic signal and is almost proportional to the change $\Delta M_{\text {perp }}$ in the perpendicular magnetization $M_{\text {perp }}$, i.e., $\Delta \theta$ is mainly attributed to the polarization rotation $\Delta \theta_{\text {MOKE }}$ induced by the magneto-optical Kerr effect. Note that the terahertz modulation of the magnetization is not caused by the magnetic field of the terahertz pulse, because the observed $\Delta \theta$ is three orders of magnitude larger than that calculated by the LLG-torque model (Supplementary Information Sec. D); rather, it is explained by the electric field of the terahertz pulse, i.e., the FKE. As shown below, in addition to this component, $\Delta \theta$ incorporates a small polarization rotation $\Delta \theta_{\text {bir }}$ induced by the birefringence, which remains owing to the small deviation from the ideal alignment of $E_{\text {probe }} / / E_{\mathrm{THz}}$, and a component of the magnetic linear dichroism $\left(\Delta \theta_{\mathrm{MLD}}\right)$, which is proportional to the square of the in-plane magnetization $\Delta M_{\text {in }}{ }^{26}$.

To quantitatively understand the magnetization dynamics, we perform the analysis using the dielectric tensor ${ }^{27}$,

$$
\widetilde{\varepsilon}=\left(\begin{array}{ccc}
\varepsilon_{x x} & \varepsilon_{x y} & 0 \\
-\varepsilon_{x y} & \varepsilon_{y y} & 0 \\
0 & 0 & \varepsilon_{z z}
\end{array}\right)
$$

Here, $x / /[1 \overline{1} 0], y / /[110]$ and $z / /[001]$ (Fig. 1a). Using this tensor and the Onsager relations, we can express $\Delta \theta_{\text {bir }}$ $\Delta \theta_{\mathrm{MOKE}}$ and $\Delta \theta_{\mathrm{MLD}}$ using $\varepsilon_{x x}, \varepsilon_{y y}, \varepsilon_{x y}$ and $\varepsilon_{z z}$, as described in Supplementary Information Sec. B. $\Delta \theta_{\mathrm{MOKE}}$, which is proportional to $\Delta M_{\text {perp }}$, is obtained by $\left(\Delta \theta_{M / /[001]}-\Delta \theta_{M / /[00 \overline{1}]}\right) / 2$, where $\Delta \theta_{M / /[001]}$ and $\Delta \theta_{M / /[00 \overline{1}]}$ denote $\Delta \theta$ measured with the magnetic field applied in the [001] and [001] directions shown in Fig. 2, respectively. Because $\Delta \theta_{\text {MOKE }}$ is influenced by not only the change in $\varepsilon_{x y}$ but also the change in $\varepsilon_{y y}$ (Supplementary Information Sec. B), we derive $-\Delta \varepsilon_{x y /} \varepsilon_{x y}$ (Fig. 3a), which is purely proportional to $\Delta M_{\text {perp }}$ divided by the initial perpendicular magnetization before the pump pulse irradiation (Supplementary Information Sec. B). Here, $\Delta \varepsilon_{x y}$ is the change in $\varepsilon_{x y}$. Figure 3a shows that $M_{\text {perp }}$ is indeed modulated by up to $1 \%$ and that it coherently follows the terahertz electric field.

For coherent magnetization control, the magnetization must tilt following the electric field of the terahertz pulse. We therefore examine the in-plane magnetization response. We derive $\Delta \theta_{\mathrm{MLD}}$, which is proportional to $\Delta M_{\mathrm{in}}^{2}$, using the relation $\Delta \theta_{\mathrm{MLD}}=\Delta \theta-\Delta \theta_{\mathrm{bir}}-\Delta \theta_{\mathrm{MOKE}}$, where $\Delta \theta_{\mathrm{bir}}$ is derived from experimental $\Delta R / R$ (Supplementary Information Sec. B). In Fig. 3b, the dynamics of $\Delta M_{\mathrm{in}}{ }^{2}$ (purple plot) show a time evolution similar to that of the perpendicular magnetization dynamics (dark blue plot in Fig. 3a), i.e., $\Delta M_{\mathrm{in}}{ }^{2}$ increases when $M_{\text {perp }}$ decreases (or when $-\Delta \varepsilon_{x y} / \varepsilon_{x y}$ increases). The geometrical calculation demonstrates that $-\Delta M_{\text {perp }}$ is proportional to $\Delta M_{\mathrm{in}}{ }^{2}$ (Supplementary Information Sec. C). Therefore, our results indicate that the magnetization is indeed tilted by the terahertz pulse. These results clearly indicate that the magnetization coherently follows the ultrafast oscillation of the electric field of the terahertz pulse via the FKE.

\section{Discussion}

We now discuss the physical mechanism of the magnetization modulation by the terahertz pulse. In the FKE, the anisotropy of the refractive index is induced by the anisotropic modulation of the band structure; owing to the 


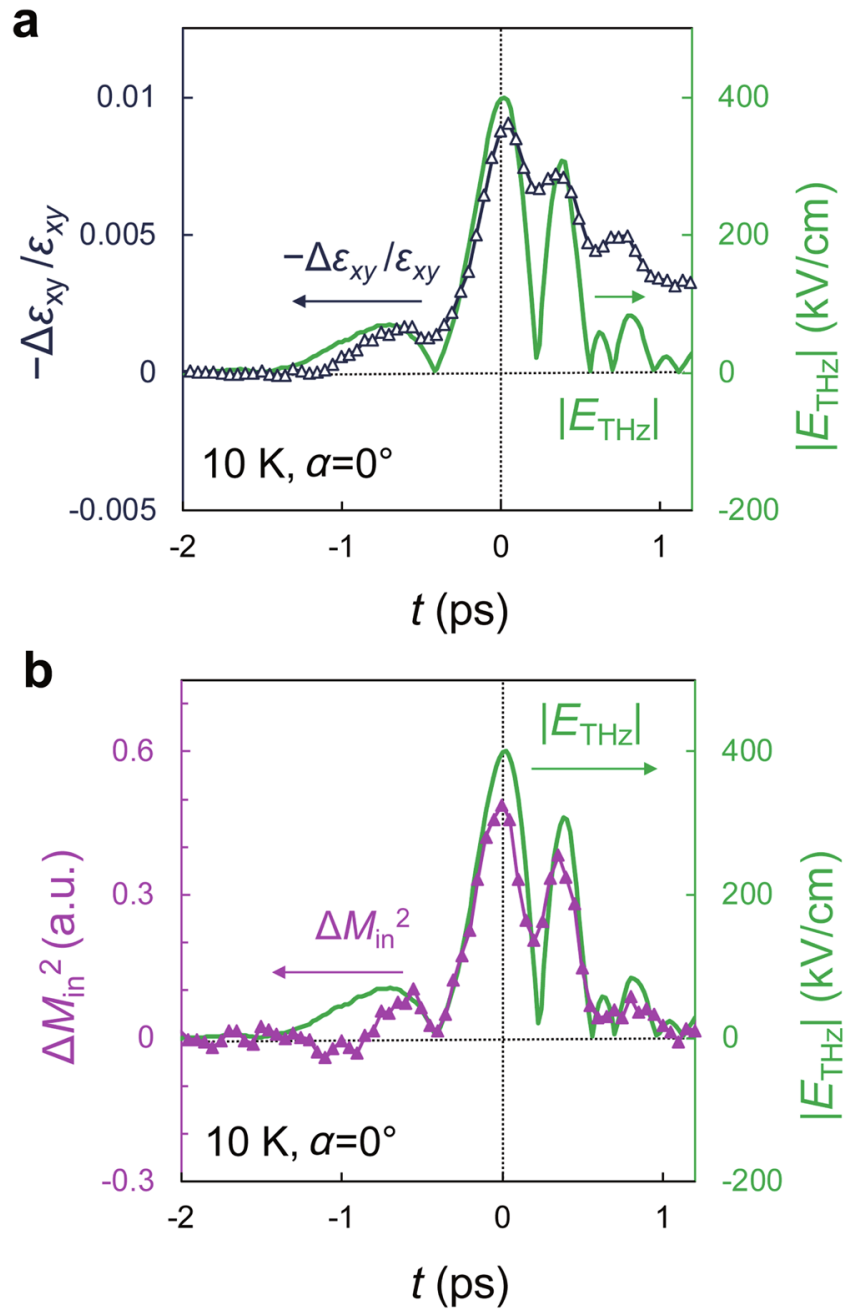

Figure 3. Magnetization modulation by $E_{\mathrm{THz}}(\mathbf{a}, \mathbf{b})$ The time evolution of $-\Delta \varepsilon_{x y} / \varepsilon_{x y}$ (dark blue plot in a) and that of $\Delta M_{\mathrm{in}}{ }^{2}$ (purple plot in $\mathbf{b}$ ). $\left|E_{\mathrm{THz}}\right|$ is shown by the green solid curve. Here, $\alpha$ is $0^{\circ}$.

application of a terahertz electric field along [110], the band structure is spatially tilted along [110] (Fig. 4a). This enables optical transitions with energies smaller than the band gap for [110]-polarized light, thus modulating the reflectivity of the material. In GaMnAs, while $E_{\mathrm{THz}}$ is applied, not only the valence band (VB) but also the electrochemical potential (EP) is tilted because carriers cannot diffuse within a few picoseconds (Fig. 4b). However, electrons can move between the VB and the impurity band (IB) (Fig. 4b), leading to the modulation in the local carrier concentration in the IB. Because ferromagnetism is induced by the double-exchange interaction between the IB holes in GaMnAs, the modulation in the carrier density of the IB induces the modulation of the magnetiza$\operatorname{tion}^{28,29}$ and its direction via spin-orbit interaction ${ }^{5}$ or the inducement of orbital angular momentum ${ }^{30}$.

At first glance, the electric field of the terahertz pulse appears to be not related to the magnetization; however, our results strongly suggest that it plays a crucial role in the terahertz response of the magnetization via the FKE. This new mechanism, the magnetization modulation via the modulation of the spatial carrier distribution induced by the electric field of light, will provide guidelines for designing materials that are suitable for coherent control of the magnetization using terahertz pulses and will provide a new approach to the ultrafast magnetization reversal.

\section{Methods}

Samples. The GaMnAs sample consists of (from top to bottom) $\mathrm{Ga}_{0,94} \mathrm{Mn}_{0.06} \mathrm{As}(20 \mathrm{~nm}) / \mathrm{In}_{0.2} \mathrm{Al}_{0.8} \mathrm{As}(500 \mathrm{~nm}$ )/GaAs $(100 \mathrm{~nm})$, which was grown on a semi-insulating GaAs (001) substrate via low-temperature molecular beam epitaxy. After growth, this sample was annealed at $180^{\circ} \mathrm{C}$ for $68 \mathrm{~h}$. The Curie temperature of the film is $125 \mathrm{~K}$. The GaMnAs film has a coercivity of $15 \mathrm{mT}$ at $10 \mathrm{~K}$. The GaAs:Be sample consists of GaAs:Be $\left(B e: 10^{19} \mathrm{~cm}^{-3}, 40 \mathrm{~nm}\right) /$ $\mathrm{In}_{0.2} \mathrm{Al}_{0.8} \mathrm{As}(500 \mathrm{~nm}) / \mathrm{GaAs}(100 \mathrm{~nm})$ grown on a semi-insulating GaAs (001) substrate.

Terahertz-pump probe measurements. The terahertz-pump probe measurements were performed using a pulsed-light source with a repetition rate of $1 \mathrm{kHz}$. Both pump and probe pulses were linearly polarized. The strong terahertz-pump pulse with a centred frequency of $1 \mathrm{THz}$, whose electric field $E_{\mathrm{THz}}$ was aligned along [110] in the film plane (Fig. 1a), was generated by tilted-pulse-front optical rectification in a $\mathrm{LiNbO}_{3}$ crystal with a tilted-pump-pulse-front scheme ${ }^{31,32}$. The measurement of $E_{\mathrm{THz}}$ is described in detail in ref. ${ }^{33}$. The terahertz 
a
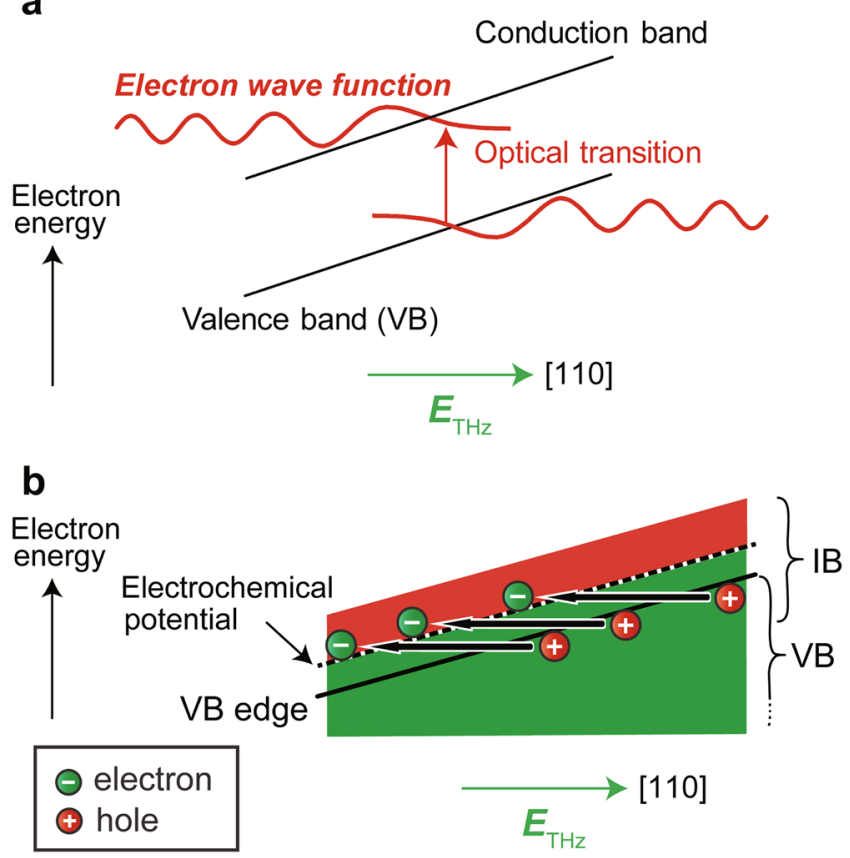

Figure 4. Magnetization modulation via the modulation of the spatial carrier distribution induced by the electric field of light. (a) Modulation of the band structure by the FKE. The band structure is spatially tilted by $E_{\mathrm{THz}}$, enabling optical transitions with an energy smaller than the band gap. The red curves represent the electron wave functions. (b) Spatial band diagram of GaMnAs while $E_{\mathrm{THz}}$ is applied. The green and red regions are filled by electrons and holes, respectively. The IB and the VB overlap. Owing to the electric field, electrons in the VB can move to the IB.

intensity was adjusted by rotating two wire-grid polarizers. The time duration of the probe pulse was $90 \mathrm{fs}$, and the wavelength was $800 \mathrm{~nm}$. The delay time of the probe pulse relative to the pump pulse was controlled by changing the length of the optical path of the probe pulse. $\Delta \theta$ was detected by a balanced detection technique using a half-wave plate, a polarizing beam splitter, a pair of balanced silicon photodiodes and a boxcar integrator. We defined the time origin $(t=0 \mathrm{ps})$ of the terahertz pulse as the time when the terahertz electric field reaches a maximum. All measurements are carried out at $10 \mathrm{~K}$.

\section{References}

1. Beaurepaire, E., Merle, J.-C., Daunois, A. \& Bigot, J.-Y. Ultrafast spin dynamics in ferromagnetic nickel. Phys. Rev. Lett. 76, 4250-4253 (1996).

2. Hohlfeld, J., Matthias, E., Knorren, R. \& Bennemann, K. H. Nonequilibrium magnetization dynamics of nickel. Phys. Rev. Lett. 78, 4861-4864 (1997).

3. Koopmans, B., van Kampen, M., Kohlhepp, J. T. \& de Jonge, W. J. Ultrafast magneto-optics in nickel: magnetism or optics? Phys. Rev. Lett. 85, 844-847 (2000).

4. van Kampen, M. et al. All-optical probe of coherent spin waves. Phys. Rev. Lett. 88, 227201 (2002).

5. Hashimoto, Y., Kobayashi, S. \& Munekata, H. Photoinduced precession of magnetization in ferromagnetic (Ga, Mn)As. Phys. Rev. Lett. 100, 067202 (2008).

6. Kirilyuk, A., Kimel, A. V. \& Rasing, T. H. Laser-induced magnetization dynamics and reversal in ferrimagnetic alloys. Rep. Prog. Phys. 76, 026501 (2013).

7. Gerrits, T. et al. Picosecond control of coherent magnetisation dynamics in permalloy thin films by picosecond magnetic field pulse shaping. J. Magn. Magn. Mater. 240, 283-286 (2002).

8. Gerrits, T., van den Berg, H. A. M., Hohlfeld, J., Bär, L. \& Rasing, T. Ultrafast precessional magnetization reversal by picosecond magnetic field pulse shaping. Nature 418, 509-512 (2002).

9. Baierl, S. et al. Nonlinear spin control by terahertz-driven anisotropy fields. Nat. Photon. 10, 715-718 (2016).

10. Shalaby, M. et al. Terahertz macrospin dynamics in insulating ferrimagnets. Phys. Rev. B 88, 140301 (2013).

11. Vicario, C. et al. Off-resonant magnetization dynamics phase-locked to an intense phase-stable terahertz transient. Nat. Photon. 7, $720-723(2013)$

12. Bonetti, S. et al. THz-driven ultrafast spin-lattice scattering in amorphous metallic ferromagnets. Phys. Rev. Lett. 117, 087205 (2016).

13. Shalaby, M., Vicario, C. \& Hauri, C. P. Simultaneous electronic and the magnetic excitation of a ferromagnet by intense THz pulses. New J. Phys. 18, 013019 (2016).

14. Chiba, D. et al. Magnetization vector manipulation by electric fields. Nature 455, 515-518 (2008).

15. Chiba, D. et al. Electrical control of the ferromagnetic phase transition in cobalt at room temperature. Nat. Mat. 10, 853-856 (2011).

16. Chiba, D., Yamanouch, M., Matsukura, F. \& Ohno, H. Electrical manipulation of magnetization reversal in a ferromagnetic semiconductor. Science 301, 943-945 (2003).

17. Chiba, D., Matsukura, F. \& Ohno, H. Electric-field control of ferromagnetism in (Ga, Mn)As. Appl. Phys. Lett. 89, 162505 (2006).

18. Sawicki, M. et al. Experimental probing of the interplay between ferromagnetism and localization in (Ga, Mn)As. Nature Phys. 6, 22-25 (2010).

19. Franz, W. Einfluß eines elektrischen feldes auf eine optische Absorptionskante. Z. Naturforsch. 13a, 484 (1958).

20. Keldysh, L. V. The effect of a strong electric field in the optical properties of insulating crystals. Soviet Physics JETP 7, 788 (1958). 
21. Kampfrath, T., Tanaka, K. \& Nelson, K. A. Resonant and nonresonant control over matter and light by intense terahertz transients. Nat. Photon. 7, 680-690 (2013).

22. Ishii, T. et al. Electronic structure near the Fermi level in the ferromagnetic semiconductor GaMnAs studied by ultrafast timeresolved light-induced reflectivity measurements. Phys. Rev. B 93, 241303 (2016).

23. Novelli, F., Fausti, D., Giusti, F., Parmigiani, F. \& Hoffmann, M. Mixed regime of light-matter interaction revealed by phase sensitive measurements of the dynamical Franz-Keldysh effect. Sci. Rep. 3, 1227 (2013).

24. Pfeifer, T., Kütt, W., Kurz, H. \& Scholz, R. Generation and detection of coherent optical phonons in germanium. Phys. Rev. Lett. 69, 3248 (1992).

25. Alping, A. \& Coldren, L. A. Electrorefraction in GaAs and InGaAsP and its application to phase modulators. J. Appl. Phys. 61, 2430 (1987).

26. Kimel, A. V. et al. Observation of giant magnetic linear dichroism in (Ga, Mn)As. Phys. Rev. Lett. 94, 227203 (2005).

27. Kahn, F. J., Pershan, P. S. \& Remeika, J. P. Ultraviolet magneto-optical properties of single-crystal orthoferrites, garnets, and other ferric oxide compounds. Phys. Rev. 186, 891-918 (1969).

28. Ohno, H. et al. Electric-field control of ferromagnetism. Nature 408, 944-946 (2000).

29. Sawicki, M. et al. Experimental probing of the interplay between ferromagnetism and localization in (Ga, Mn)As. Nat. Phys. 6, 22-25 (2010).

30. Matsuda, T. \& Munekata, H. Mechanism of photoexcited precession of magnetization in (Ga, Mn)As on the basis of time-resolved spectroscopy. Phys. Rev. B 93, 075202 (2016).

31. Hebling, J., Almási, G., Kozma, I. Z. \& Kuhl, J. Velocity matching by pulse front tilting for large area THz-pulse generation. Opt. Express 10, 1161-1166 (2002).

32. Hirori, H., Doi, A., Blanchard, F. \& Tanaka, K. Single-cycle terahertz pulses with amplitudes exceeding $1 \mathrm{MV} / \mathrm{cm}$ generated by optical rectification in $\mathrm{LiNbO}_{3}$. Appl. Phys. Lett. 98, 091106 (2011).

33. Miyamoto, T., Yada, H., Yamakawa, H. \& Okamoto, H. Ultrafast modulation of polarization amplitude by terahertz fields in electronic-type organic ferroelectrics. Nat. Commun. 4, 2586 (2013).

\section{Acknowledgements}

This work was partly supported by Grants-in-Aid for Scientific Research (No. 26249039, 18H03860, ISHO1011, 16H02095), the Project for Developing Innovation Systems of MEXT, Spintronics Research Network of Japan, and CREST, Japan Science and Technology Agency (Grant No. JPMJCR1661). T.I., H.Y. and T.K. were supported by the Japan Society for the Promotion of Science (JSPS) through the Program for Leading Graduate Schools (MERIT). T.I., H.Y. and T.K. thank the JSPS Research Fellowship Program for Young Scientists for support.

\section{Author Contributions}

T.I. and H.Y. conceived the experiment. T.K. and T.I. grew and characterized the samples. H.Y., T.M. and N.K. constructed the terahertz-pump optical-probe systems. T.I. and H.Y. performed the measurements. T.I. analysed the data. H.O., M.T. and S.O. coordinated the study. T.I. and S.O. wrote the paper with inputs from all authors.

\section{Additional Information}

Supplementary information accompanies this paper at https://doi.org/10.1038/s41598-018-25266-2.

Competing Interests: The authors declare no competing interests.

Publisher's note: Springer Nature remains neutral with regard to jurisdictional claims in published maps and institutional affiliations.

(c) (i) Open Access This article is licensed under a Creative Commons Attribution 4.0 International Cicense, which permits use, sharing, adaptation, distribution and reproduction in any medium or format, as long as you give appropriate credit to the original author(s) and the source, provide a link to the Creative Commons license, and indicate if changes were made. The images or other third party material in this article are included in the article's Creative Commons license, unless indicated otherwise in a credit line to the material. If material is not included in the article's Creative Commons license and your intended use is not permitted by statutory regulation or exceeds the permitted use, you will need to obtain permission directly from the copyright holder. To view a copy of this license, visit http://creativecommons.org/licenses/by/4.0/.

(c) The Author(s) 2018 